\title{
Cardiac sympathectomy and spinal cord stimulation attenuate reflex-mediated norepinephrine release during ischemia preventing ventricular fibrillation
}

\author{
Jeffrey L. Ardell,, ${ }^{1,2,3,4}$ Robert D. Foreman, ${ }^{5}$ J. Andrew Armour, ${ }^{1,2}$ and Kalyanam Shivkumar ${ }^{1,2,3,6}$ \\ 'UCLA Cardiac Arrhythmia Center, UCLA Health System, Los Angeles, California, USA. ${ }^{2}$ Neurocardiology Research Program \\ of Excellence and ${ }^{3}$ Molecular Cellular and Integrative Physiology, UCLA, Los Angeles, California, USA. ${ }^{4}$ Department \\ of Biomedical Sciences, East Tennessee State University, Johnson City, Tennessee, USA. ${ }^{5}$ Department of Physiology, \\ University of Oklahoma Health Science Center, Oklahoma City, Oklahoma, USA. \\ ${ }^{6}$ Neuroscience Interdepartmental Programs, UCLA, Los Angeles, California, USA.
}

The purpose of this study was to define the mechanism by which cardiac neuraxial decentralization or spinal cord stimulation (SCS) reduces ischemia-induced ventricular fibrillation (VF). Direct measurements of norepinephrine (NE) levels in the left ventricular interstitial fluid (ISF) by microdialysis, in response to transient (15-minute) coronary artery occlusion (CAO), were performed in anesthetized canines. Responses were studied in animals with intact neuraxes and were compared with those in which the intrathoracic component of the cardiac neuraxes (stellate ganglia) or the intrinsic cardiac neuronal (ICN) system was surgically delinked from the central nervous system and those with intact neuraxes with preemptive SCS (T1-T3). With intact neuraxes, animals with exaggerated NE release due to CAO were at increased risk for VF. During CAO, there was a $152 \%$ increase in NE when the neuraxes were intact compared with $114 \%$ following stellate decentralization and $16 \%$ following ICN decentralization. During SCS, CAO NE levels increased by $59 \%$. Risk for CAO-induced VF was $38 \%$ in controls, $8 \%$ following decentralization, and $11 \%$ following SCS. These data indicate that ischemia-related afferent neuronal transmission differentially engages central and intrathoracic sympathetic reflexes and amplifies sympathoexcitation. Differences in regional ventricular NE release are associated with increased risk for VF. Surgical decentralization or SCS reduced NE release and VF.

Conflict of interest: The University of California (UCLA) has patents developed by our group in the areas of cardiac catheter technology, embolism prevention technology, minimally invasive methods for cardiac interventions, cardiac neural diagnostics, and therapeutics.

Copyright: () 2019, American Society for Clinical Investigation.

Submitted: July 9, 2019 Accepted: October 23, 2019 Published: December 5, 2019.

Reference information: /CI Insight. 2019;4(23):e131648. https://doi.org/10.1172/jici. insight.131648.

\section{Introduction}

Acute myocardial ischemia (MI) can initiate lethal cardiac arrhythmias that are responsible for sudden cardiac death (SCD) (1). Local metabolic factors, including ionic shifts $(1,2)$, contribute to the creation of an arrhythmogenic substrate for arrhythmias. Vulnerability to MI arrhythmia induction is also powerfully modulated by the autonomic neural networks (3-5). Cardiac afferent sensory transduction initiates reflexes within the cardiac nervous system (6) that ultimately regulate efferent sympathetic output to the heart. These reflexes result in the release of norepinephrine (NE) at the nerve terminals into the cardiac interstitium (7-10). This reflex-mediated NE release can be highly arrhythmogenic at a time when the myocardium itself is highly susceptible to abnormal impulse formation and propagation $(3,5,11)$. Pharmacological approaches to mitigate these consequences have focused on adrenergic receptor blockade, which is an established therapy primarily targeted at the cardiac end effectors (12). The antiarrhythmic efficacy of such pharmacological therapies to reduce SCD has limitations and addresses only one aspect of the pathophysiological mechanism leading to sympathoexcitation $(3,5,13)$. The contribution of cardiac innervation to the risk of ventricular fibrillation (VF), especially via the transduction of ischemia by neural afferents that cause reflex mediated sympathoexcitation, is not well understood.

The cardiac nervous system comprises a series of feedback control circuits, which are fundamental to dynamic cardiac control in health and disease (14-16). New avenues for neuraxial therapeutic interventions can be understood with this framework (17-19). Neuromodulation therapies are targeted at specific 
Table 1. Hemodynamic response to transient coronary artery occlusion

\begin{tabular}{|c|c|c|c|c|}
\hline & Heart rate (beats/min) & LV DP (mmHg) & LV SP (mmHg) & LV dp/dt (mmHg/s) \\
\hline \multicolumn{5}{|l|}{ Intact } \\
\hline Baseline & $134.7 \pm 4.0$ & $2.0 \pm 0.7$ & $115.6 \pm 5.7$ & $3175.3 \pm 215.8$ \\
\hline Reperfusion & $135.1 \pm 4.7$ & $1.6 \pm 0.6$ & $109.6 \pm 8.5$ & $2602.4 \pm 160.1^{\mathrm{A}}$ \\
\hline \multicolumn{5}{|c|}{ Stellate ganglia decentralized } \\
\hline Baseline & $105.0 \pm 7.7$ & $3.8 \pm 0.8$ & $105.5 \pm 6.5$ & $2307.4 \pm 235.3$ \\
\hline \multicolumn{5}{|c|}{ Intrinsic cardiac ganglia decentralized } \\
\hline Baseline & $98.9 \pm 5.6$ & $2.7 \pm 0.6$ & $108.5 \pm 4.4$ & $2447.3 \pm 171.0$ \\
\hline LAD CAO & $103.2 \pm 7.5$ & $5.6 \pm 0.7^{A}$ & $95.9 \pm 6.4^{A}$ & $2000.8 \pm 283.9^{A}$ \\
\hline Reperfusion & $99.0 \pm 5.7$ & $3.3 \pm 0.6$ & $98.8 \pm 5.4^{\mathrm{A}}$ & $2144.1 \pm 175.7$ \\
\hline \multicolumn{5}{|l|}{ Intact T1-T3 SCS } \\
\hline
\end{tabular}

Hemodynamic data derived in response to transient coronary artery occlusions (CAO) and reperfusion in each of the 4 experimental groups. ${ }^{A} P \leq 0.05$ versus baseline, repeated-measure ANOVA with Holm-Sidak method for pairwise multiple comparison. SP, systolic pressure; DP, diastolic pressure.

"nexus" points of the cardiac nervous system such as the intrinsic cardiac nervous system (ICNS) (17), extracardiac sympathetic ganglia (19-21), spinal cord (22-24), or vagosympathetic trunk (25-28). These therapies have demonstrated myocardial salvage $(24,27,29)$ and efficacy in arrhythmia control $(20,21,23)$. Neuraxial stabilization is further exemplified in the transplanted heart, which contains a functional ICNS $(30,31)$, and is decentralized from the higher levels of the nervous system. In this setting, VF is rarely seen in transplanted hearts - even in the setting of acute ischemia (32). The mechanistic basis of these clinical observations, especially why there is a reduction in sympathoexcitation with cardiac neural decentralization, is not well understood. We sought to examine the effects of stepwise decentralization of the heart and spinal cord stimulation (SCS) in separate sets of experiments with the aid of direct neurotransmitter measurements of NE levels in the left ventricle interstitial fluid (ISF) space with the microdialysis technique.

We hypothesized that neural reflexes initiated by cardiac sensory transduction of ventricular ischemia are responsible for amplification of sympathoexcitation and resultant enhancement of regional cardiac NE levels leading to VF. We also hypothesized that stabilization of intrathoracic reflex processing (by SCS or surgical decentralization of the intrathoracic aspects of the cardiac nervous system from higher centers) can mitigate this amplification of sympathoexcitation during myocardial/ischemia reperfusion, thereby reducing regional ventricular NE release and, thus, decreasing the potential for VF.

\section{Results}

Hemodynamic indices. Table 1 summarizes the hemodynamic data recorded before, during, and after transient occlusion of the left anterior descending (LAD) coronary artery in each of the 4 animal groups studied. With the exception of the SCS neuromodulation group, LAD occlusion initiated minimal changes in heart rate. It decreased left ventricular (LV) systolic pressure and LV $+\mathrm{dP} / \mathrm{dt}$. It also increased LV end-diastolic pressure. While SCS by itself did not alter baseline hemodynamic indices, it did mitigate depressions in $\mathrm{LV}+\mathrm{dP} / \mathrm{dt}$ associated with transient LAD occlusion.

$V F$. In untreated control animals, transient (15 minutes) occlusion of the LAD coronary artery was associated with VF in 38\% of the animals (group 1) (Figures 1 and 2). This generally occurred within 3 minutes of reperfusion onset (Figure $2 \mathrm{~A}$, innervation intact) and was associated with surge in LV ISF catecholamine levels $(109.8 \% \pm 25.0 \%$ change over baseline for survivors vs. $458.3 \% \pm 63.6 \%$ change over baseline for animals that went into VF; $P \leq 0.001)$. In a subset of animals with intact innervation $(n=11)$, the circumflex artery was occluded, with VF onset primarily occurring 4-6 minutes into occlusion (Figure 2A, red trace). For the animals that experienced VF during circumflex CAO, the 


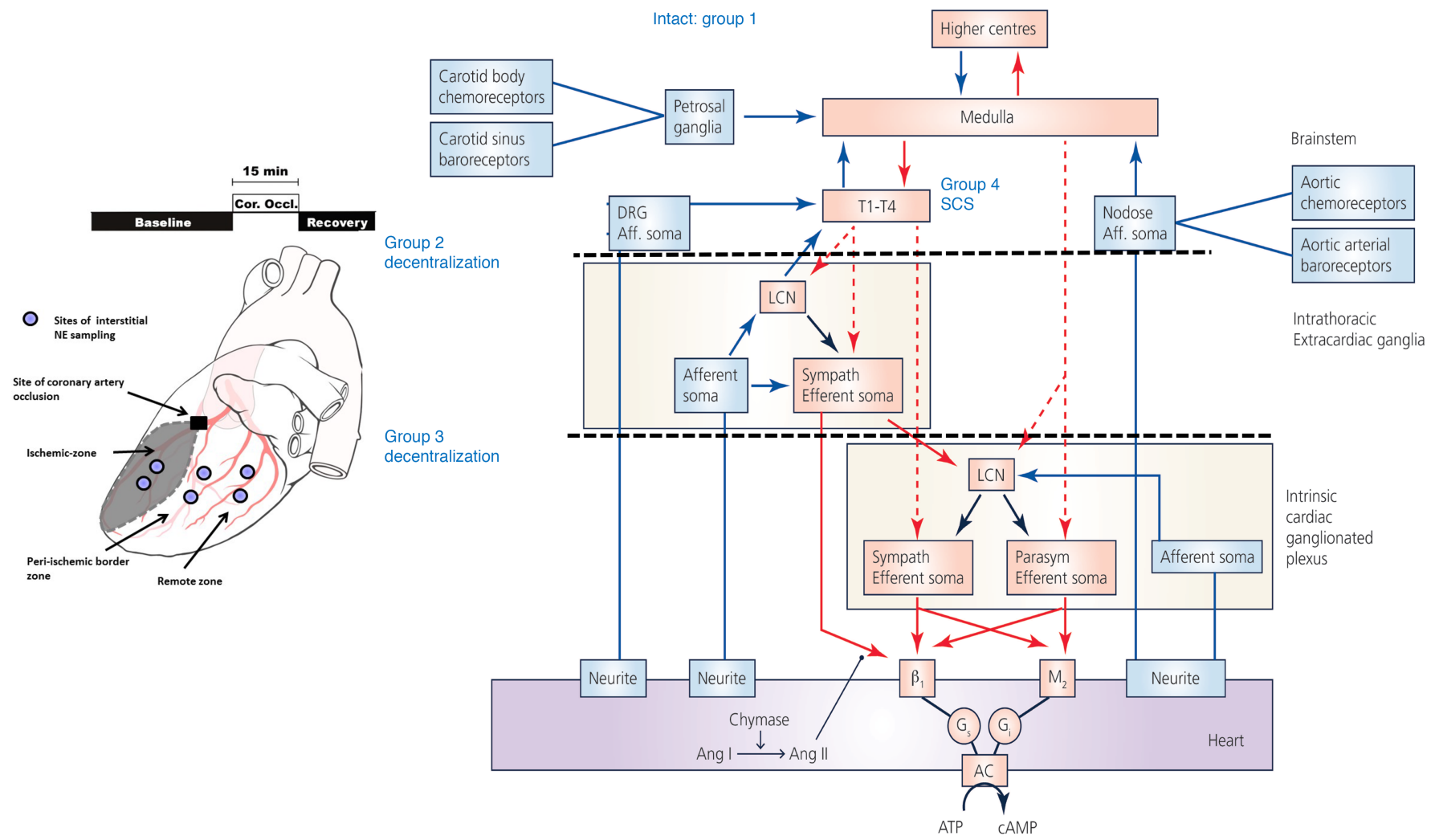

Figure 1. Experimental design. Summary of experimental protocol of coronary occlusion and sites of microdialysis for norepinephrine (NE) collection. The left anterior descending coronary artery (LAD) is shown. The levels of decentralization as related to primary elements of the neural hierarchy for cardiac control are shown. Responses in animals with (a) intact neuraxes (group 1) were compared with those in which the (b) intrathoracic component of the cardiac neuraxes was surgically disconnected from the central nervous system and (c) those with intact neuraxes subjected to SCS (group 4; T1-T3 spinal level). For group 2 decentralization, the cervical vagosympathetic nerve trunks were transected and all connections from the cardiac nervous system to stellate ganglia were transected. For group 3 decentralization, all connections to (efferent) and from (afferent) the heart were surgically interrupted. LCN, local circuit neurons; Sympath, sympathetic; Parasym, parasympathetic; Aff, afferent; T, thoracic spinal segment; C, cervical spinal segment; DRG, dorsal root ganglia; $\mathrm{G}_{\mathrm{s}}$ and $\mathrm{G}_{\mathrm{i}}$, stimulatory and inhibitory guanine nucleotide binding protein.

ischemic zone had catecholamines levels twice that of resistant animals $(2037 \pm 161 \mathrm{pg} / \mathrm{ml} \mathrm{vs} .1255$ $\pm 210 \mathrm{pg} / \mathrm{ml}, P=0.028$ ) during that ischemic event. Acute decentralization of the intrathoracic nervous system from the influences of central neurons reduced the potential for ischemia-induced SCD to $8 \%$ of control (occurring in 1 of 12 animals so tested, Figure 2B) (groups 2 and 3). Preemptive SCS also reduced the incidence of CAO-induced VF in neurally intact preparations (1 of 9 animal during $\mathrm{SCS}+\mathrm{CAO}$ so studied; Figure 2C) (group 4). From group 4, 2 of 5 animals randomized to CAO first experienced SCD/VF at reperfusion. In group 4 animals, randomized first to SCS $+\mathrm{CAO}$ and then, 1 hour later, to $\mathrm{CAO}$ alone, 5 of 6 animals survived the first ischemic challenge (c.f., in the presence of SCS). In response to the second (sham SCS) CAO, animals exhibited a $40 \%$ potential for SCD/VF. As detailed in the next section, the reduction in VF potential by sequential decentralization or active SCS was associated with mitigation of NE release during ischemia and reperfusion phases.

Local LV interstitial NE release with an intact neuraxis. Regional ventricular NE liberation was greatest during transition states (i.e., baseline to occlusion or occlusion to reperfusion) (Figure 3, A and B). When these data were subgrouped retrospectively according to whether animals survived the ischemic insult (VF resistant) versus those that did not survive (VF susceptible), animals that died suddenly demonstrated enhanced NE levels within the ischemic zone during CAO and enhanced NE levels during reperfusion in all areas of the LV sampled (Figure 3, C and D, and Figure 4A). In intact preparations that survived the transient ischemic challenge, regional LV ISF NE increased in reflex response to LAD CAO/reperfusion - with progressively increasing contents when comparing normal with ischemic zones (703.3 \pm $74.0 \mathrm{pg} / \mathrm{ml}$ to $1426.0 \pm 156.7 \mathrm{pg} / \mathrm{ml}$, normal zone; $799.1 \pm 57.8 \mathrm{pg} / \mathrm{ml}$ to $1933.1 \pm 183.8 \mathrm{pg} / \mathrm{ml}$, ischemic zone; $P=0.318$ at baseline and $P=0.047$ during LAD CAO between zones). 
A Innervation Intact

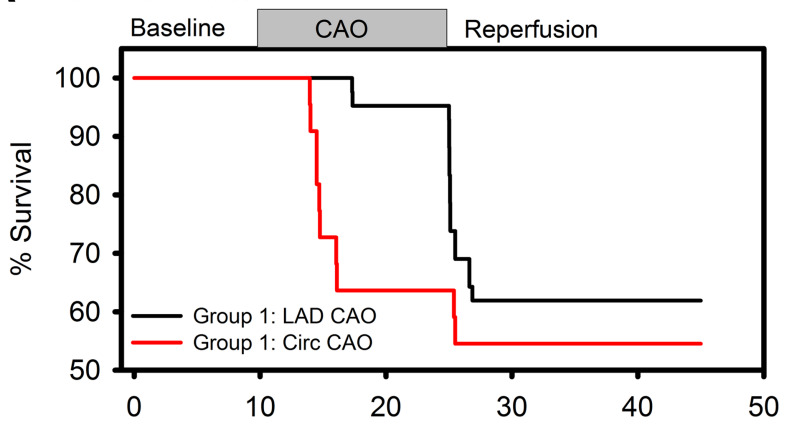

B Stellate decent (Group 2), ICN decent (Group 3)

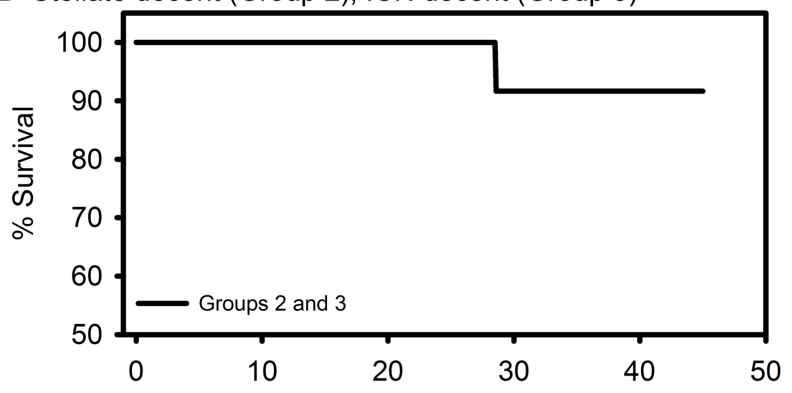

C Pre-emptive SCS

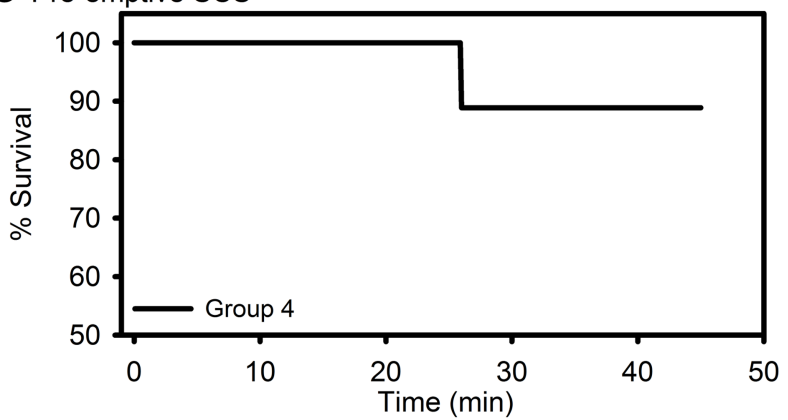

Figure 2. Survival following coronary artery occlusion and reperfusion. (A) Survival curves in animals with intact innervation in response to transient LAD or circumflex CAO. Surgical decentralization of intrathoracic ganglia of the cardiac nervous system (B) and preemptive T1-T3 spinal cord stimulation (SCS) (C) reduced mortality (percentage mortality) to 15-minute occlusion (gray bar) of the left anterior descending coronary artery (CAO) as compared with untreated controls. The intrathoracic decentralization curve includes both group 2 and group $3 . P<$ 0.05 , log rank-sum test LAD CAO intact vs. LAD CAO decentralized. $P<0.05$, proportion of animals alive after 45 minutes of each intervention, group 4 versus group 1 .

CAO-induced LV ISF NE following decentralization of the intrathoracic nervous system. When the intrathoracic elements of the cardiac nervous system were surgically disconnected from the central nervous system (stellate ganglia decentralized and cervical vagosympathetic trunks cut bilaterally), transient CAO still reflexly increased NE contents in all $3 \mathrm{LV}$ regions (ischemic, border, and normal zones) (Figure $4 \mathrm{~B}$ ). However, such increase was reduced by approximately $25 \%$ across all zones when compared with data obtained in the intact neural state preparations. Following decentralization of the intrinsic cardiac neuronal (ICN), leaving it as the sole reflex controller of neuronal release, CAO reflex-induced release of NE into the nonischemic and border zones was virtually eliminated. Residual ischemia-induced NE release was identified within the ischemic zone (Figure 4C). Following ICN decentralization, no evidence of enhanced NE release was evident upon reperfusion in any of the zones evaluated.

CAO-induced LV ISF NE following thoracic SCS. Electrical stimulation of the dorsal columns of the high thoracic spinal cord (T1-T3) blunted MI-induced reflex-induced release of NE into the ISF in all LV regions tested $(149.3 \% \pm 33.9 \%$ vs. $38.4 \% \pm 14.7 \%$ normal zone, $P \leq 0.015 ; 206.8 \% \pm 34.1 \%$ vs. $80.1 \pm 10.8, P$ $\leq 0.001$ ischemic zone; percentage change from basal levels) (Figure 5). Preemptive SCS had no effect on basal hemodynamics but did mitigate the depression in LV contractility that was identified in control states in response to LAD CAO (Table 1). In contrast, in controls in timed experiments, similar regional ISF NE release and evoked hemodynamic responses were identified during repeat CAO (data not shown).

\section{Discussion}

The major findings of this study are as follows. Afferent sensory transduction of ventricular ischemia results in a reflex-mediated amplification of sympathoexcitation. The augmented NE release due to afferents engaging higher levels of the neuraxis and concomitant regional dispersion in NE release in the heart was associated 
A
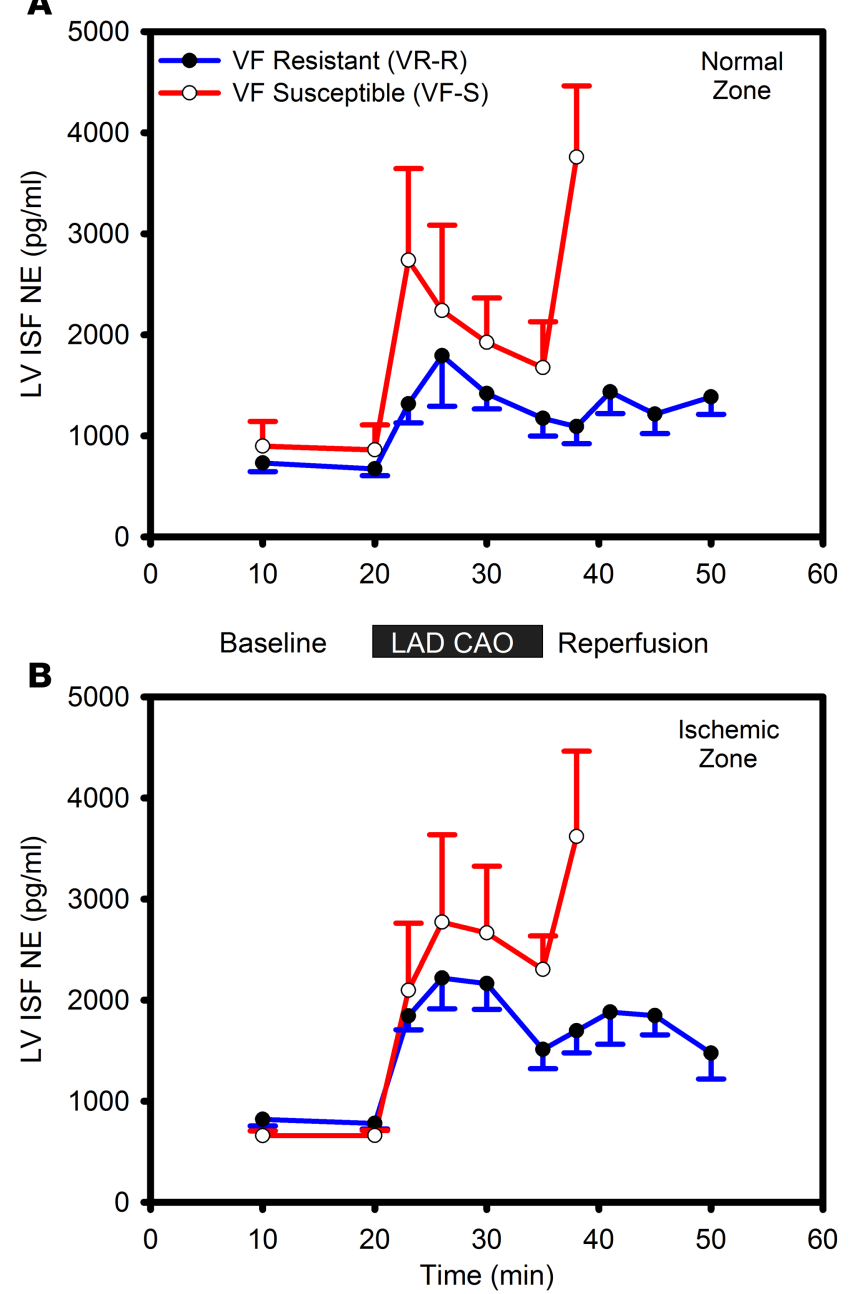

C

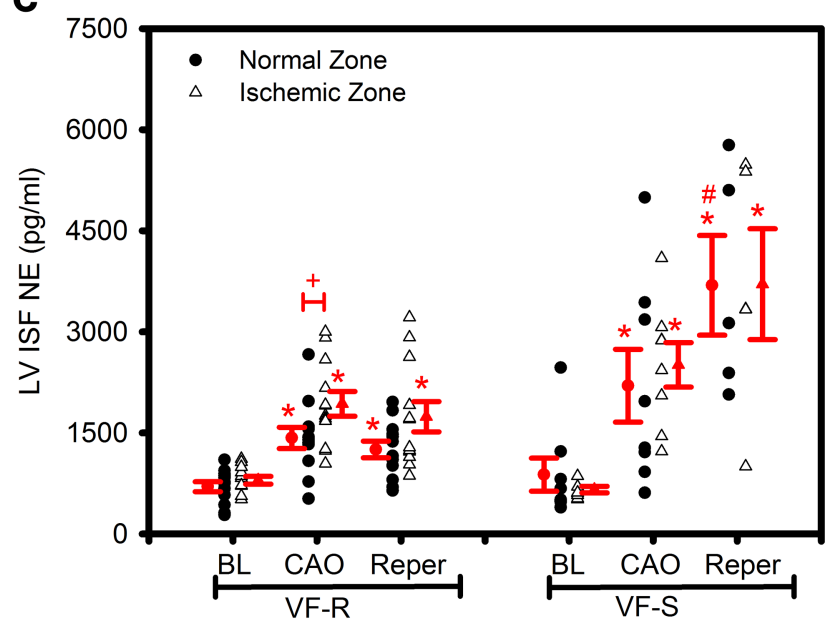

D

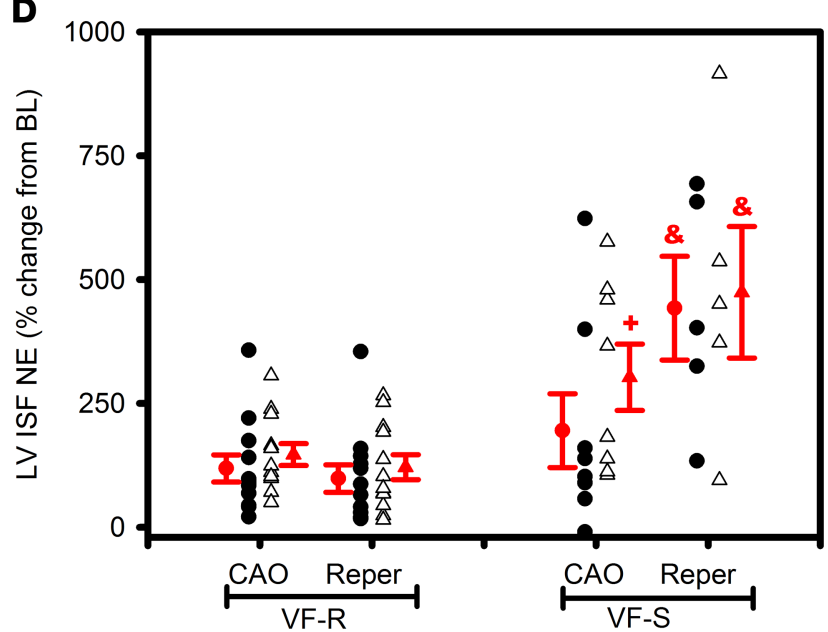

Figure 3. LV interstitial fluid NE VF-resistant versus VF-susceptible subjects. Changes induced in the interstitial levels of norepinephrine (NE) (mean \pm SEM) in response to 15 -minute LAD coronary artery occlusion (CAO) in LV tissues of the normal zone (A) compared with changes in the ischemic zone (B). Data are subdivided into those animals that tolerated the transient ischemic insult (VF resistant [VF-R]; $n=12$ ) versus those that did not (VF susceptible [VF-S]; $n=8$ ). (A and B) Temporal changes in LV ISF NE from baseline to reperfusion phases (VF-S, red lines; VF-S, blue lines). (C) Average LV ISF NE levels during baseline (BL), CAO, and reperfusion (repeated-measure ANOVA, with Holm-Sidak method for multiple pairwise comparison). (D) Percentage change from baseline during CAO and reperfusion phases for VF-R versus VF-S animals (Kruskal-Wallis ANOVA on ranks, with Dunn's methods for pairwise multiple comparisons). ${ }^{*} P \leq 0.01$ vs. baseline within subgroup (VF-R or VS-S); $P \leq 0.04$ CAO vs. reperfusion within subgroup, between group comparisons: ${ }^{+} P \leq 0.05$ VF-R vs. VF-S; ${ }^{P} P \leq 0.01$ VF-R vs. VF-S.

increased incidence of VF. Progressive "delinking" of the heart from more rostral aspects of the neuraxis attenuates the amplification of sympathoexcitation by the higher regions. Neuraxial modulation using SCS mitigated MI reflex-induced augmentation of NE release; VF was reduced with preservation of contractile function.

Cardiac neurotransmission. The heart has a rich sensory innervation that transduces multiple physiological events that are transmitted to various levels of the neuraxis (Figure 1 and refs. 17, 33, 34). Afferent neural traffic is transmitted to the intrinsic neural ganglionated plexus in the heart, stellate/middle cervical ganglia, the spinal cord (via dorsal root ganglia) with relays to higher centers via the spinoreticular track, and by way of the vagosympathetic trunk to the nodose ganglia with neuronal projections to the nucleus tractus solitaries $(10,30,34,35)$. This afferent information is processed by the ICNS, stellate/middle cervical ganglia, spinal cord, and higher centers to initiate the reflexes that ultimately result in activation of sympathetic efferent postganglionic neurons within intrathoracic extracardiac and intrinsic cardiac ganglia $(17,33,36)$. This reflex-induced sympathoexcitation modulates cardiac chronotropy, dromotropy, inotropy, and lusitropy $(37,38)$.

Integrated control of cardiac indices by adrenergic efferent postganglionic neurons can be investigated locally by assessment of regional catecholamine release (39-41). In this study ISF levels of NE were determined in normal (remote), ischemic, and border zone regions of the left ventricle in reflex response to transient, 
A

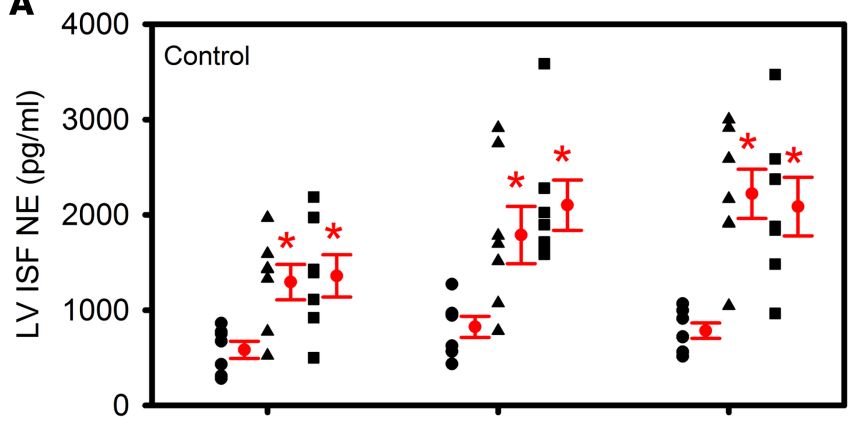

B
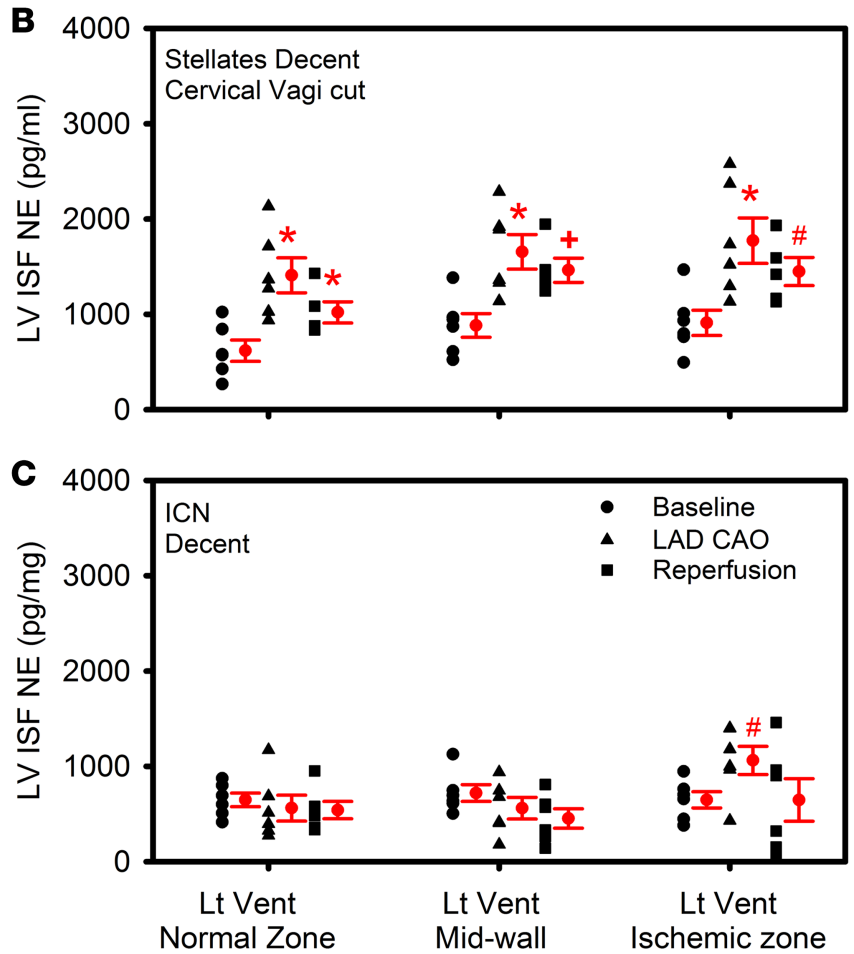

Figure 4. LV norepinephrine release following neuraxial decentralization. LV ISF norepinephrine (NE) levels in the ventral region perfused by the circumflex coronary artery (normal zone), LV midwall region (border zone), and the region perfused by the LAD coronary artery (ischemic zone) at baseline, during LAD $C A O$, and reperfusion. These data were derived from animals with intact neuraxes (A, control, group1, $n=7$ ) versus those in which the intrathoracic nervous system was acutely decentralized (stellate decent, stellate ganglia decentralized and cervical vagosympathetic complexes cut) (B, group 2, $n=6$ ) versus those in which the intrinsic cardiac nervous system (ICN) was surgically decentralized from all extracardiac neuronal inputs (C, ICN Decent, group 3, $n=6$ ). ${ }^{*} P \leq 0.01$ from baseline; ${ }^{+} P \leq 0.03$ from baseline; $\# P \leq 0.05$ from baseline, repeated-measure ANOVA, with Holm-Sidak method for pairwise multiple comparison.

regional LV ischemia; levels of NE measured within the ISF are reflective of neuronal release, reuptake, and diffusion $(39,40,42)$. This ISF-NE index of regional sympathetic activity was assessed in animals with (a) intact neuraxes, as compared with those in which (b) the intrathoracic component of the cardiac neuronal hierarchy was surgically disconnected from the central nervous system or (c) those with intact neuraxes that had been preemptively treated with SCS (T1-T3 spinal levels). The results show that processing of cardiac afferents at each level of the neuraxis results in amplification of sympathoexcitation, as evidenced by higher NE release, and that targeted neuromodulation has the capacity to blunt such reflexes while preserving basal function.

Hierarchy of cardiac neural control. Hierarchy for cardiac control functions as a series of nested feedback loops. Control is regulated by 3 major neuronal levels: (a) the ICNS, (b) extracardiac intrathoracic (stellate, middle cervical, and mediastinal) ganglia, and (c) the central nervous system (spinal cord, brainstem, and higher centers) $(17,43-45)$. These 3 levels are capable of independent and interdependent reflex actions for control of regional cardiac function $(30,35,46,47)$. The 2 lower level reflex circuits that are contained with the thoracic cavity (ICNS and stellate/middle cervical/ mediastinal ganglia) are "cardiocentric" and are primarily concerned with dynamic control of regional cardiac indices $(22,30,48,49)$. Our study shows that their efferent output is delineated by level of the hierarchy involved $(10,17,36)$. ICNS-evoked responses to transient MI reflect cardiocentric control restricted primarily to the ischemic zone and results in the least degree of amplification of sympathoexcitation $(49,50)$. In contrast, intrathoracic-extracardiac (stellate/middle cervical ganglia-dependent) reflex responses to the ischemic stress involved coordination of adrenergic neurons that released $\mathrm{NE}$ throughout the left ventricle in reflex response to a focal ischemic stress $(22,41,42)$. 


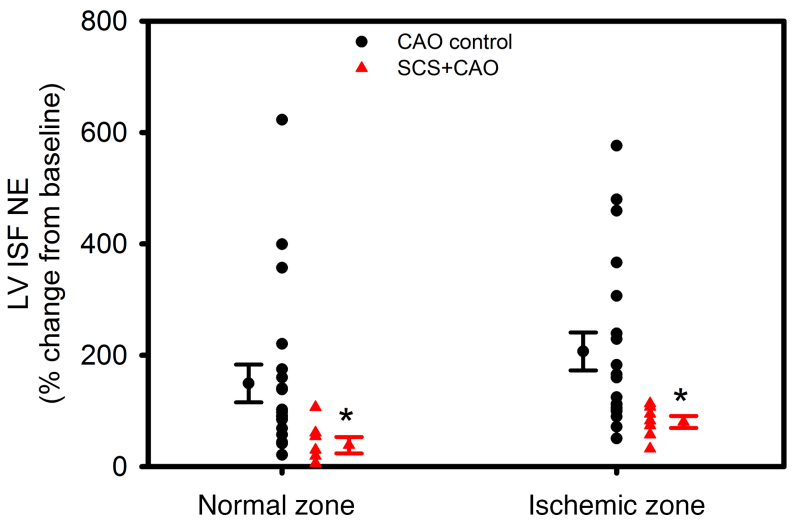

Figure 5. Coronary artery occlusion-induced LV ISF NE changes following thoracic spinal cord stimulation. Changes induced in regional ventricular ISF NE levels in normal versus ischemic zones in reflex response to transient (15 minutes) LAD coronary artery occlusion (CAO) without (control, $n=20$ ) versus with preemptive neuromodulation (SCS, $n=7$ ). Note that SCS reduced ischemia-induced reflex NE release in both the normal and ischemic zones. ${ }^{*} P \leq 0.02 \mathrm{SCS}+\mathrm{CAO}$ vs. untreated CAO, Kruskal-Wallis ANOVA on ranks, with Dunn's methods for pairwise multiple comparisons.

Centrally mediated effects. Superimposed upon these intrathoracic (peripheral) reflexes are those mediated by the central nervous system. Centrally mediated reflexes are essential to controlling not only cardiac function but also blood flow demands throughout the entire body $(37,51,52)$. In response to normal physiological stressors (e.g., orthostatic stress and dynamic exercise), changes in cardiovascular-related afferent inputs, along with somatoautonomic interactions, adjust cardiac output to meet whole-body blood flow demand $(37,51,52)$. Conversely, pathophysiological stressors, such as acute MI, have the potential to disrupt such control, sometimes with lethal consequences $(3,4,11,51)$. Our data show the highest degree of amplification of sympathoexcitation is seen when the higher centers are engaged. Our findings also highlight the importance of measures such as sedation and general anesthesia for the management of ventricular arrhythmia storm (53).

Effect of MI. MI presents a novel, major stressor to the cardiac neuraxis (51). It is known that visceral sensory information is integrated at multiple levels within the central nervous system, including the spinal cord $(10,44,54)$ and brainstem $(36,37,43)$. From an integrated control perspective, afferent input (ischemia) elicits a differential response from the peripheral cardiocentric reflex loop (32, 50, 51 ) as compared with the response from central neural networks $(9,10,34,43)$. Specifically, our data show that the reflex response of the higher centers to sensory inputs from the ischemic myocardium is inherently proarrhythmic (amplification of sympathoexcitation); however, when the central sites are delinked, peripheral reflexes are less arrhythmogenic and are accompanied by a lower release of NE in the myocardium. It should be noted that there is still release within the ischemic zone, even after ICN decentralization. This could be "cardiocardiac reflex" mediated or could reflect the local effects of extracellular $\mathrm{K}^{+}$following myocyte death.

Neuraxial modulation/clinical relevance. The results shown here are directly relevant to dorsal (high thoracic) SCS $(55,56)$ and decentralization of the intrathoracic aspects of the cardiac nervous system from the central nervous system (as seen with bilateral stellate ganglionectomy) (19-21). Previous data have demonstrated that SCS is effective in stabilizing reflex processing within extracardiac sympathetic ganglia (22) and the intrinsic cardiac ganglionated plexus (57), while reducing the apoptotic potential of ischemic stressed cardiomyocytes (24). Our results demonstrate that SCS blunted neuronal NE release into the LV interstitium with an associated reduced risk for SCD.

Classically, neuronal control of sympathetic outflows to the heart has been considered to be primarily under central neuronal command, with peripheral ganglia functioning solely as efferent neuronal relay stations $(37,58)$. Extrapolating from that conceptual framework, decentralization of the intrathoracic elements of the cardiac nervous system would be expected to abolish all MI-induced catecholamine release, except for local effects mediated directly on adrenergic efferent postganglionic nerve terminals located within the ischemic zone $(42,59)$. In contrast, our data show that following decentralization of intrathoracic reflex control from the influences of central neurons, effective coordination of local catecholamine release across the ventricle is maintained (Figure 4). In fact, the disparity in such 
release between the risk zone and normally perfused tissues actually decreased. At the same time, such a reduction in NE dispersion conferred a mortality benefit (Figure 2). These results directly relate to the clinical setting of intractable ventricular tachycardia (VT), where stellate ganglia decentralization is effective in the management of VT storm (19-21).

Limitations. This study, being conducted in anesthetized animals, does not take into account the depressor effects of anesthetic agents on reflex regulation of cardiac NE release. However, general anesthesia does not fully inhibit centrally driven proarrhythmic events, as evidenced by the efficacy of thoracic epicardial anesthesia and stellate ganglionectomy in the clinical setting $(20,53)$. We did not directly record from intrathoracic extracardiac and intrinsic cardiac neurons concomitant with cardiac indices in this study. However, previous studies done by our group are consistent with the reduced adrenergic outflow noted in this study with SCS or decentralization $(22,31,50,57)$. Finally, transection of the vagus also removes afferent neural signals from the heart, which could have also regulated NE release and interrupted parasympathetic efferent projections to the heart, which mitigates the potential for peripheral sympathetic/parasympathetic interactions (60-64). Preconditioning effects could also play a role in these situations. To minimize this, we performed repeat occlusions in animals that did not have VF and responses were similar. For group 4, in the animals that experienced SCS +CAO first $(n=6), 5$ survived the first transient ischemic insult, with 2 more of those animals experiencing VF in response to the second untreated CAO event. This $40 \%$ mortality ( 3 of 5) matches the overall mortality for the untreated group 1 (38\% mortality). This argues against preconditioning being a major factor for group 4 (SCS).

Conclusion. This study indicates that regional ventricular ischemia activates central and intrathoracic reflexes (including those confined to the ICNS) that control cardiac sympathetic efferent postganglionic neurons, which release NE into the ventricular interstitium. As summarized in the Graphical Abstract, the magnitude of regional release in response to the afferent transduction of ventricular ischemia is augmented and amplified by afferents engaging the higher levels of the neuraxis (stellates, spinal cord, and the brain). Delinking the heart from each higher level of the neuraxis (by surgery or via bioelectronically) was protective against the transient stress of CAO. This is analogous to the electrical stability of the transplanted human heart $(32,65)$. These data provide a mechanistic basis to understand why procedures such as cardiac sympathectomy (19-21) and SCS (23) are effective in arrhythmia control.

\section{Methods}

\section{Model}

Thirty-four mongrel dogs (Hodgins Kennel) of either sex, weighing between 20 and $30 \mathrm{~kg}$, were used in these studies.

\section{Surgical preparation}

Anesthesia was induced with sodium thiopental $(6 \mathrm{mg} / \mathrm{kg})$ and maintained with isofluorane $(2 \%$, inhalation therapy). A 5-Fr catheter transducer (Mikro-tip, Millar) was inserted into the LV chamber via the right femoral artery to measure LV pressure and LV $+\mathrm{dp} / \mathrm{dt}$. Aortic pressure was monitored via a 5-Fr catheter transducer placed via the left femoral artery. Heart rate was monitored via a lead II electrocardiogram. A bilateral thoracotomy (fourth interspace) was performed. For the subsequent induction of transient regional ventricular ischemia, after exposing the heart, a pericardial cradle was formed and silk ligatures were placed around the LAD coronary artery $1.5 \mathrm{~cm}$ from its origin (or the proximal circumflex coronary artery for the experiments on that territory). All hemodynamic data were digitized (Cambridge Electronic Design power 1401 acquisition system with Spike 2 software) for subsequent off-line analysis.

\section{Neuromodulation treatment groups}

Surgical decentralization of the intrathoracic cardiac nervous system. Four groups were used to evaluate the central-peripheral interactions in reflex control of LV-ISF NE levels in response to transient CAO, each targeting a different level of the cardiac neuraxes (Figure 1). Figure 1 summarizes the experimental design for selective neuraxial modulation/isolation. Group 1 ( $n=11 \mathrm{dogs}$ ) had an intact cardiac neuraxis and served as the untreated control for the other groups. For group 2 ( $n=6$ dogs), all neural connections between the stellate ganglia and spinal cord were severed bilaterally and both cervical 
vagosympathetic trunks transected. These surgical procedures decentralized neurons in stellate, middle cervical, mediastinal, and intrinsic cardiac ganglia from the central nervous system (66). For group 3 ( $n$ $=6$ dogs), the stellate, middle cervical, and mediastinal ganglia were excised surgically bilaterally, along with their entire intrathoracic extracardiac vagosympathetic complex (30). This procedure removed all extracardiac neuronal connections (afferent and efferent) to the ICNS, leaving it as the sole neuronal controller of cardiac indices $(17,30)$.

$S C S$. In group $4(n=11)$, following induction of anesthesia, using techniques described previously (22, 55), the epidural space was accessed via a Tuohy needle, and a 4-pole electrode (Octrode, Advanced Neuromodulation Systems) was advanced rostrally in the epidural space to the T1-T3 spinal cord level (Figure 1). The tip of the lead was positioned under fluoroscopy with the multipole electrode placed midway between the midline and epidural region overlying left dorsal funiculus. T1-T3 SCS was delivered at a frequency of $50 \mathrm{~Hz}$, a duration of 200 milliseconds, and a current intensity of $90 \%$ motor threshold (MT), with the rostral pole as cathode. This level of stimulation corresponds to that used clinically to treat angina (55) and in both small and large animals to modulate intrathoracic autonomic reflex processing $(22,57)$. Following SCS implant, the animal was rotated to the decubitus position, the chest opened, and the animals were instrumented as detailed above. SCS MT levels were verified prior to and following completion of experimental protocol.

\section{Ventricular microdialysis}

Regional ventricular ISF NE levels were quantified using the microdialysis technique (40-42). Six microdialysis probes were placed into the LV wall. As illustrated in Figure 2, 2 probes were inserted into the anterior wall region that was perfused by the LAD coronary artery (ischemic zone) and 2 were inserted into the posterior-lateral LV wall. The remaining 2 probes were placed midway between the 2 other sets, a region that represented the border zone.

In each group, regional ventricular ischemia was induced by a 15-minute period of LAD CAO. Repeat occlusions were separated by at least 1 hour. In groups 2 and 3, surgical decentralization of intrathoracic versus intrinsic cardiac neurons was completed at least 1 hour prior to placement of the microdialysis probes. Dialysate was collected separately at baseline, during 3-minute intervals throughout CAO, and for 15 minutes following CAO. Concurrent changes in cardiovascular hemodynamic indices were recorded. In group 4, at least 1 hour separated arterial occlusions. All NE levels were assessed while the animals were still in sinus rhythm; we stopped collections that would have gone into the period of VF to avoid confounding effects of increasing NE levels due to VF-induced sympathetic surge.

The treatment order (preemptive SCS+CAO vs. CAO) was randomized. In the SCS group, T1-T3 SCS was delivered at $50 \mathrm{~Hz}$, with a 200-millisecond duration and at a current intensity of $90 \% \mathrm{MT}(0.79 \pm 0.16$ $\mathrm{mA}$ ). SCS was initiated 15 minutes before CAO began and lasted for 36 minutes. SCS MT was checked periodically and was found to not vary significantly from initial levels.

\section{Biochemical analyses}

Ventricular dialysate fluid collected from the $3 \mathrm{LV}$ zones (normal, border, and ischemic tissues) was analyzed for NE content during baseline and during each successive challenge. LV ISF NE concentrations were determined using radioenzymatic assay, as we described previously $(40,67)$. Derived ISF levels for NE are reflective of regional sympathetic efferent postganglionic neuronal release, local catecholamine reuptake, and diffusion from measurement sites.

\section{Statistical analyses}

Data are presented as mean \pm SEM. One-way ANOVA with post hoc comparison (Holm-Sidak method) was used to compare hemodynamic and NE concentration data derived at baseline, LAD CAO, and reperfusion. If data failed the normality test (Shapiro-Wilk), Kruskal-Wallis 1-way ANOVA on ranks was used with post hoc comparison (Dunn's method). A $P$ value of less than or equal to 0.05 was considered significant.

\section{Study approval}

All experiments were performed in accordance with the guidelines for animal experimentation described in the Guide for the Care and Use of Laboratory Animals (National Academy Press, 2011). The Institutional Animal Care and Use Committee of East Tennessee State University approved these experiments. 


\section{Author contributions}

JLA, RDF, JAA, and KS contributed to production and presentation of the data and approved the final version of the manuscript.

\section{Acknowledgments}

This study was supported by NIH grants HL71830 (to JLA), HL084261 (to KS), and OT20D023848 (to KS and JLA).

Address correspondence to: Jeffrey L. Ardell or Kalyanam Shivkumar, UCLA Cardiac Arrhythmia Center, 100 Medical Plaza, Suite 660, UCLA Health System, Los Angeles, California 90095, USA. Phone: 310.825.0417; Email: jardell@mednet.ucla.edu (JLA). Phone: 310.206.6433; Email: kshivkumar@mednet.ucla.edu (KS).

1. Janse MJ, Wit AL. Electrophysiological mechanisms of ventricular arrhythmias resulting from myocardial ischemia and infarction. Physiol Rev. 1989;69(4):1049-1169.

2. Shivkumar K, Deutsch NA, Lamp ST, Khuu K, Goldhaber JI, Weiss JN. Mechanism of hypoxic K loss in rabbit ventricle. J Clin Invest. 1997;100(7):1782-1788.

3. Fukuda K, Kanazawa H, Aizawa Y, Ardell JL, Shivkumar K. Cardiac innervation and sudden cardiac death. Circ Res. 2015;116(12):2005-2019.

4. Schwartz PJ, La Rovere MT, Vanoli E. Autonomic nervous system and sudden cardiac death. Experimental basis and clinical observations for post-myocardial infarction risk stratification. Circulation. 1992;85(1 Suppl):I77-I91.

5. Shen MJ, Zipes DP. Role of the autonomic nervous system in modulating cardiac arrhythmias. Circ Res. 2014;114(6):1004-1021.

6. Malliani A, Schwartz PJ, Zanchetti A. A sympathetic reflex elicited by experimental coronary occlusion. Am J Physiol. 1969;217(3):703-709.

7. Malliani A, Lombardi F, Pagani M. Functions of afferents in cardiovascular sympathetic nerves. J Auton Nerv Syst. 1981;3(24):231-236.

8. Malliani A, Recordati G, Schwartz PJ. Nervous activity of afferent cardiac sympathetic fibres with atrial and ventricular endings. The Journal of physiology. 1973;229(2):457-469.

9. Foreman RD. Mechanisms of cardiac pain. Annu Rev Physiol. 1999;61:143-167.

10. Wang HJ, Wang W, Cornish KG, Rozanski GJ, Zucker IH. Cardiac sympathetic afferent denervation attenuates cardiac remodeling and improves cardiovascular dysfunction in rats with heart failure. Hypertension. 2014;64(4):745-755.

11. Billman GE. A comprehensive review and analysis of 25 years of data from an in vivo canine model of sudden cardiac death: implications for future anti-arrhythmic drug development. Pharmacol Ther. 2006;111(3):808-835.

12. European Heart Rhythm Association, et al. ACC/AHA/ESC 2006 guidelines for management of patients with ventricular arrhythmias and the prevention of sudden cardiac death: a report of the American College of Cardiology/American Heart Association Task Force and the European Society of Cardiology Committee for Practice Guidelines (Writing Committee to Develop Guidelines for Management of Patients With Ventricular Arrhythmias and the Prevention of Sudden Cardiac Death). J Am Coll Cardiol. 2006;48(5):e247-e346.

13. Al Chekakie MO. Traditional heart failure medications and sudden cardiac death prevention: a review. J Cardiovasc Pharmacol Ther. 2013;18(5):412-426.

14. Ardell JL, et al. Translational neurocardiology: preclinical models and cardioneural integrative aspects. J Physiol (Lond). 2016;594(14):3877-3909.

15. Ardell JL, Armour JA. Neurocardiology: Structure-Based Function. Compr Physiol. 2016;6(4):1635-1653.

16. Shivkumar K, et al. Clinical neurocardiology defining the value of neuroscience-based cardiovascular therapeutics. $J$ Physiol (Lond). 2016;594(14):3911-3954.

17. Armour JA. Potential clinical relevance of the "little brain" on the mammalian heart. Exp Physiol. 2008;93(2):165-176.

18. Lopshire JC, Zipes DP. Device therapy to modulate the autonomic nervous system to treat heart failure. Curr Cardiol Rep. 2012;14(5):593-600.

19. Vaseghi M, et al. Cardiac sympathetic denervation in patients with refractory ventricular arrhythmias or electrical storm: intermediate and long-term follow-up. Heart Rhythm. 2014;11(3):360-366.

20. Ajijola OA, et al. Bilateral cardiac sympathetic denervation for the management of electrical storm. J Am Coll Cardiol. 2012;59(1):91-92.

21. Schwartz PJ. Cardiac sympathetic denervation to prevent life-threatening arrhythmias. Nat Rev Cardiol. 2014;11(6):346-353.

22. Ardell JL, Cardinal R, Vermeulen M, Armour JA. Dorsal spinal cord stimulation obtunds the capacity of intrathoracic extracardiac neurons to transduce myocardial ischemia. Am J Physiol Regul Integr Comp Physiol. 2009;297(2):R470-R477.

23. Issa $\mathrm{ZF}$, et al. Thoracic spinal cord stimulation reduces the risk of ischemic ventricular arrhythmias in a postinfarction heart failure canine model. Circulation. 2005;111(24):3217-3220.

24. Southerland EM, et al. Preemptive, but not reactive, spinal cord stimulation mitigates transient ischemia-induced myocardial infarction via cardiac adrenergic neurons. Am J Physiol Heart Circ Physiol. 2007;292(1):H311-H317.

25. De Ferrari GM. Vagal stimulation in heart failure. J Cardiovasc Transl Res. 2014;7(3):310-320.

26. Premchand RK, et al. Autonomic regulation therapy via left or right cervical vagus nerve stimulation in patients with chronic heart failure: results of the ANTHEM-HF trial. J Card Fail. 2014;20(11):808-816.

27. Shinlapawittayatorn $\mathrm{K}$, et al. Low-amplitude, left vagus nerve stimulation significantly attenuates ventricular dysfunction and infarct size through prevention of mitochondrial dysfunction during acute ischemia-reperfusion injury. Heart Rhythm. 
2013;10(11):1700-1707.

28. Vanoli E, De Ferrari GM, Stramba-Badiale M, Hull SS, Foreman RD, Schwartz PJ. Vagal stimulation and prevention of sudden death in conscious dogs with a healed myocardial infarction. Circ Res. 1991;68(5):1471-1481.

29. Liao SY, et al. Remodelling of cardiac sympathetic re-innervation with thoracic spinal cord stimulation improves left ventricular function in a porcine model of heart failure. Europace. 2015;17(12):1875-1883.

30. Ardell JL, Butler CK, Smith FM, Hopkins DA, Armour JA. Activity of in vivo atrial and ventricular neurons in chronically decentralized canine hearts. Am J Physiol. 1991;260(3 Pt 2):H713-H721.

31. Murphy DA, et al. The heart reinnervates after transplantation. Ann Thorac Surg. 2000;69(6):1769-1781.

32. Vaseghi M, et al. Supraventricular tachycardia after orthotopic cardiac transplantation. J Am Coll Cardiol. 2008;51(23):2241-2249.

33. Ardell JL. Intrathoracic Neuronal Regulation of Cardiac Function. In: Armour JA, Ardell JL, eds. Basic and Clinical Neurocardiology. New York, New York, USA: Oxford University Press; 2004:118-52.

34. Harper RM, Kumar R, Macey PM, Ogren JA, Richardson HL. Functional neuroanatomy and sleep-disordered breathing: implications for autonomic regulation. Anat Rec (Hoboken). 2012;295(9):1385-1395.

35. Armour JA, Collier K, Kember G, Ardell JL. Differential selectivity of cardiac neurons in separate intrathoracic autonomic ganglia. Am J Physiol. 1998;274(4):R939-R949.

36. Zucker IH, Patel KP, Schultz HD. Neurohumoral stimulation. Heart Fail Clin. 2012;8(1):87-99.

37. Jänig W. The Integrative Action of the Autonomic Nervous System: Neurobiology of Homeostasis. New York, New York, USA: Cambridge University Press; 2006.

38. Randall WC, Armour JA. Regional vagosympathetic control of the heart. Am J Physiol. 1974;227(2):444-452.

39. Eisenhofer G, et al. Cardiac sympathetic nerve function in congestive heart failure. Circulation. 1996;93(9):1667-1676.

40. Farrell DM, et al. Angiotensin II modulates catecholamine release into interstitial fluid of canine myocardium in vivo. Am J Physiol Heart Circ Physiol. 2001;281(2):H813-H822.

41. Kawada T, et al. Regional difference in ischaemia-induced myocardial interstitial noradrenaline and acetylcholine releases Auton Neurosci. 2007;137(1-2):44-50.

42. Lameris TW, et al. Time course and mechanism of myocardial catecholamine release during transient ischemia in vivo. Circulation. 2000;101(22):2645-2650.

43. Andresen MC, Kunze DL, Mendelowitz D. Central Nervous System Regulation of the Heart. In: Armour JA, Ardell JL, eds. Basic and Clinical Neurocardiology. New York, New York, USA: Oxford University Press; 2004:187-219.

44. Foreman RDD, DeJongste MJL, Linderoth B. Integrative Control of Cardiac Function by Cervical and Thoracic Spinal Neurons. In: Armour JA, Ardell JL, eds. Basic and Clinical Neurocardiology. New York, New York, USA: Oxford University Press; 2004:153-86.

45. Kember G, Armour JA, Zamir M. Neural control of heart rate: the role of neuronal networking. J Theor Biol. 2011;277(1):41-47.

46. McAllen RM, Salo LM, Paton JF, Pickering AE. Processing of central and reflex vagal drives by rat cardiac ganglion neurones: an intracellular analysis. J Physiol (Lond). 2011;589(Pt 23):5801-5818.

47. Waldmann M, Thompson GW, Kember GC, Ardell JL, Armour JA. Stochastic behavior of atrial and ventricular intrinsic cardiac neurons. J Appl Physiol. 2006;101(2):413-419.

48. Armour JA. Activity of in situ stellate ganglion neurons of dogs recorded extracellularly. Can J Physiol Pharmacol. 1986;64(2):101-111.

49. Beaumont E, et al. Network interactions within the canine intrinsic cardiac nervous system: implications for reflex control of regional cardiac function. J Physiol (Lond). 2013;591(18):4515-4533.

50. Huang MH, Ardell JL, Hanna BD, Wolf SG, Armour JA. Effects of transient coronary artery occlusion on canine intrinsic cardiac neuronal activity. Integr Physiol Behav Sci. 1993;28(1):5-21.

51. Kember G, Armour JA, Zamir M. Neural control hierarchy of the heart has not evolved to deal with myocardial ischemia. Physiol Genomics. 2013;45(15):638-644.

52. Zucker IH, Gilmore JP. Reflex Control of the Circulation. Boca Raton, Florida, USA: CRC Press; 1991

53. Bourke T, et al. Neuraxial modulation for refractory ventricular arrhythmias: value of thoracic epidural anesthesia and surgical left cardiac sympathetic denervation. Circulation. 2010;121(21):2255-2262.

54. Ding X, et al. Modulation of cardiac ischemia-sensitive afferent neuron signaling by preemptive C2 spinal cord stimulation: effect on substance P release from rat spinal cord. Am J Physiol Regul Integr Comp Physiol. 2008;294(1):R93-101.

55. Andréll P, et al. Long-term effects of spinal cord stimulation on angina symptoms and quality of life in patients with refractory angina pectoris--results from the European Angina Registry Link Study (EARL). Heart. 2010;96(14):1132-1136.

56. Tse HF, et al. Thoracic Spinal Cord Stimulation for Heart Failure as a Restorative Treatment (SCS HEART study): first-in-man experience. Heart Rhythm. 2015;12(3):588-595.

57. Armour JA, et al. Long-term modulation of the intrinsic cardiac nervous system by spinal cord neurons in normal and ischaemic hearts. Auton Neurosci. 2002;95(1-2):71-79.

58. Langley G. The Autonomic Nervous System. Cambridge, United Kingdom: Cambridge University Press; 1921.

59. Kurz T, Richardt G, Hagl S, Seyfarth M, Schömig A. Two different mechanisms of noradrenaline release during normoxia and simulated ischemia in human cardiac tissue. J Mol Cell Cardiol. 1995;27(5):1161-1172.

60. Qin C, Farber JP, Linderoth B, Shahid A, Foreman RD. Neuromodulation of thoracic intraspinal visceroreceptive transmission by electrical stimulation of spinal dorsal column and somatic afferents in rats. J Pain. 2008;9(1):71-78.

61. Chandler MJ, Zhang J, Qin C, Foreman RD. Spinal inhibitory effects of cardiopulmonary afferent inputs in monkeys: neuronal processing in high cervical segments. J Neurophysiol. 2002;87(3):1290-1302.

62. Southerland EM, et al. Activated cranial cervical cord neurons affect left ventricular infarct size and the potential for sudden cardiac death. Auton Neurosci. 2012;169(1):34-42.

63. McGuirt AS, Schmacht DC, Ardell JL. Autonomic interactions for control of atrial rate are maintained after SA nodal parasympathectomy. Am J Physiol. 1997;272(6 Pt 2):H2525-H2533.

64. Levy MN, Martin PJ. Neural Control of the Heart. In: Berne RM, ed. The Heart. Bethesda, Marylabnd, USA: American Physiological Society; 1979:581-620. Handbook of Physiology: Section 2: The Cardiovascular System; vol 1. 
65. Vaseghi M, et al. Mode and mechanisms of death after orthotopic heart transplantation. Heart Rhythm. 2009;6(4):503-509.

66. Armour JA. Synaptic transmission in the chronically decentralized middle cervical and stellate ganglia of the dog. Can J Physiol Pharmacol. 1983;61(10):1149-1155.

67. Hankes GH, et al. Beta1-adrenoceptor blockade mitigates excessive norepinephrine release into cardiac interstitium in mitral regurgitation in dog. Am J Physiol Heart Circ Physiol. 2006;291(1):H147-H151. 